\title{
Number of severe bleeding complications according to classification used: Is unified classification of bleeding complications really necessary?
}

\author{
Magdalena Sionova ${ }^{1}$, Peter Blasko ${ }^{2}$, Stepan Jirous ${ }^{3}$, David Vindis ${ }^{4}$, \\ Richard Rokyta ${ }^{3}$, Lenka Posch ${ }^{5}$, Zuzana Motovska ${ }^{1}$ \\ ${ }^{1}$ Third Medical Faculty, Charles University and \\ University Hospital Kralovske Vinohrady, Prague, Czech Republic \\ ${ }^{2}$ University Hospital and Faculty of Medicine, Ostrava, Czech Republic \\ ${ }^{3}$ University Hospital and Faculty of Medicine, Pilsen, Czech Republic
}

${ }^{4}$ University Hospital and Faculty of Medicine and Dentistry, Palacky University, Olomouc, Czech Republic

${ }^{5}$ First Medical Faculty, Charles University, Prague, Czech Republic

\begin{abstract}
Background: To compare the number of severe periprocedural bleeding complications from the total number of bleeding complications associated with diagnostic selective coronary angiography or percutaneous coronary intervention (PCI) when using different classifications (TIMI, GUSTO, PLATO, BARC) and to relate these classifications to real hemodynamic status of evaluated patients.
\end{abstract}

Methods: We analyzed data from 106 patients who underwent invasive procedure for ischemic heart disease (selective coronary angiography/PCI) and suffered from any type of bleeding complication.

Results: The number of bleeding according to impacts on hemodynamic status and consequent treatment shows that $54.7 \%$ of all bleedings did not need any specific therapy. Bleeding leading to death, hemorrhagic shock, hemodynamic instability, administration of blood transfusion, surgical procedure and local treatment occurred in 6.6\%, 1.9\%, 5.7\%, 14.2\%, 2.8\%, and $14.2 \%$, respectively. The results comparing bleeding classifications demonstrate that the rate of severe bleeding complications may increase up to 4 times when different classifications are used on the same group of patients (TIMI 9.4\%, GUSTO 15.1\%, PLATO $39.2 \%$ and BARC 35.9\%). The power of association between severe bleeding determined by these classifications and real hemodynamic compromise using Kendall's tau-c correlation is -0.4106 (95\% CI -0.599 to -0.222), -0.5355 (95\% CI -0.718 to-0.353), -0.5513 (95\% CI-0.729 to -0.374) and-0.7552 (95\% CI-0.897 to-0.612) for TIMI, GUSTO, PLATO and BARC, respectively. Conclusions: The data show significant dependence of percentage of severe periprocedural bleeding complications on selected classification. The strongest association between severe bleeding and real hemodynamic status was found for BARC classification as this classification seems to be promising for future general use. (Cardiol J 2015; 22, 6: 665-674)

Key words: ischemic heart disease, bleeding complications, classification of bleeding

Address for correspondence: Zuzana Motovska, MD, PhD, Cardiocentre, University Hospital Kralovske Vinohrady, Šrobárova 50, Prague 100 34, Czech Republic, tel: + 420267163 760, fax: +420 267163 763, e-mail: zuzana.motovska@fnkv.cz

Received: 27.04.2015 Accepted: 03.07.2015 


\section{Introduction}

The treatment of patients with acute coronary syndrome (ACS), as well as those with stable form of ischemic heart disease (IHD) has developed rapidly in the last several decades. Invasive procedures in cardiology, e.g. selective coronary angiography (CAG) as a diagnostic method and percutaneous coronary intervention (PCI) as a therapeutic method have become part of a routine practice. With continuous improvement in technique of CAG and PCI, with new materials used and mainly with development of new types of more effective antiplatelet and anticoagulant drugs, the number of death rates according to ischemic events and periprocedural ischemic complications including ischemic stroke and heart failure has decreased to minimum and almost reached boundary for further reduction $[1,2]$. On the other hand, older people and patients with wider range of comorbidities undergo invasive procedure as a therapeutic and very often it is the only diagnostic method for all forms of IHD [3].

All factors mentioned above are responsible for increased risk of bleeding complications in connection with invasive procedures [4]. As bleeding complications are associated with worse short-term, as well as long-term prognosis for patients suffering from them, they become a noticeable medical and economical problem and so they are becoming more in a point of interest for all experts and clinicians [5-7]. The incidence of bleeding complication in clinical trials varies ranging from $2.0 \%$ to $17.6 \%$ differing according to population evaluated, cardiac catheterization technique, pharmacotherapy used and many others $[4,5,8-10]$. In most of recent large randomized clinical trials, the rate of bleeding complications is used as a safety indicator for every new procedure in invasive cardiology and the rate of bleeding complications indicates safety of all newly introduced antiplatelet or anticoagulant drugs $[11,12]$.

Furthermore, in current era of evidence based medicine, it is then very important to compare acquired data from all those provided clinical trials with each other. In association with bleeding complications, the comparability is now affected with wide number of different classifications that have been used for evaluation of those complications in last decades [13]. Each classification has been tailored according to study design meaning different outcomes are evaluated for in-hospital mortality of patients with ACS requiring rescue PCI than in classification for long term follow up evaluating wide range of bleeding events (including superficial bleeding, e.g. petechia).

This variability in bleeding classifications used across clinical trials led us to compare the most often used classifications. The aim was to find out how different outcomes may develop when various classifications are used in one patient group. Then we correlate evaluation of each of those classifications with real seriousness of bleeding expressed by hemodynamic compromise and required treatment in context of finding that the clinical criteria are more important for sequential clinical outcomes.

\section{Methods}

We analyzed periprocedural bleeding complications in a group of patients who developed bleeding in association with CAG or PCI. Ethics Committees of participating institutions approved the study protocol. Patients were included in the study after signing an informed consent for participation. No exclusion criteria were applied for study participation.

Basic demographic data, medical history, cardiovascular risk factors, major comorbidities, clinical status and laboratory findings were recorded. Medical history was obtained from patients' medical documentation or personal interview. Clinical evaluation was performed during admission to the hospital and then relevant changes of clinical status were noted during hospitalization. Blood count was measured in all patients with a special emphasis on level of hemoglobin and hematocrit. The kidney function was monitored using creatinine clearance received by Cockroft-Gault formula. Also factors related directly to invasive procedure including number of affected vessels, administered antiplatelet or anticoagulant drugs and type of arterial access (femoral or radial) were observed.

All bleeding complications that occurred relating to CAG or PCI were precisely described in every patient from multiple perspectives. In all patients, we observed the real hemodynamic compromise (according to the criteria listed below) and therapy required when any type of bleeding occurred. The hemodynamic status and its changes, the necessity of intensive care unit, administration of any blood transfusions as well as provided therapy were thoroughly monitored. Also, the localization of each bleeding was observed by using various imaging techniques where needed and applicable (computed tomography [CT], ultrasound [US]). 
With all these collected data mentioned above we were able to process number of bleeding complications according to different criteria. We evaluated number of these complications according to i) hemodynamic compromise of the patients and subsequent need of treatment ii) localization of the bleeding. For evaluation hemodynamic compromise and subsequent treatment we established 7 consecutive groups according to severity of bleeding: 1) bleeding with no need of treatment, 2) with local therapy needed, 3) with blood transfusion administration, 4) with surgical revision, 5) bleeding leading to hemodynamic instability (defined as decrease in blood pressure affecting the function of any organ requiring intravenous solution or any supportive drug administration which do not meet criteria for hemorrhagic shock), 6) bleeding leading to hemorrhagic shock (defined as bleeding meeting at least 3 following criteria: with systolic blood pressure $<90 \mathrm{~mm} \mathrm{Hg}$, tachycardia $>120 / \mathrm{min}$, anuria, tachypnea $>30 / \mathrm{min}$, quantitative or qualitative alteration of consciousness), 7) fatal bleeding. Then we applied on this group of patients 4 different classifications of bleeding complications that are standardly used for evaluating data from large randomized cardiovascular clinical trials Thrombolysis in Myocardial Infarction (TIMI), Global Use of Strategies to Open Occluded Arteries (GUSTO), Platelet Inhibition and Patient Outcomes (PLATO) and Bleeding Academic Research Consortium (BARC) [12, 14-16]. The TIMI and GUSTO classification were chosen as representatives of the basic and simple classifications with opposite monitored values (laboratory vs. clinical data). Detailed criteria of each classification are displayed in Supplementary Table S1 and Supplementary Table S2. The PLATO was selected as an example of independent classification with fusion of both laboratory and clinical data and the BARC classification as a representative of the newest and highly elaborated bleeding classification. Due to these classifications we observed percentage of severe complications, as for better comparability were marked as severe in PLATO together life-threatening and other severe bleeding and in BARC classification together number of bleeding from group $3 \mathrm{a}-5 \mathrm{~b}$. Then we were able to compare the differences in rate of severe complications in each group.

Finally, we made a correlation between the seriousness of bleeding according to hemodynamic compromise with each bleeding classification separately. Each of the 4 representative classifications provides an ordinal scale. The scales given by clas- sifications TIMI, GUSTO and PLATO distinguish 3 degrees of bleeding severity, while BARC provides a more complex scale. The hemodynamic status is also described by an ordinal scale which distinguishes 7 levels defined above. As the relations between each of the 4 classifications and patients' real hemodynamic status were to be compared, the most suitable statistic to use was Kendal's tau-c. Kendal's tau-c coefficient ranges from -1 to 1 meaning strong negative and strong positive association, respectively. The values close to -1 signify the higher degree of particular classification the worse hemodynamic status, therefore the strongest correlation. The values close to 1 , on the contrary, indicate increase in severity of bleeding due to bleeding classification with conversely improving hemodynamic status. The values close to 0 can be interpreted as no association between bleeding severity and the hemodynamic compromise [17]. The Kendal's coefficients were obtained with the use of STATISTICA, version 10.

\section{Results}

The study population consists of 106 patients who developed bleeding complication in association with invasive procedure performed for any form of IHD from 5 cardio centers from Czech Republic (Prague, Pilsen, Olomouc, Zlin, Ostrava) between 2006 and 2012. Only patients with all data required for analysis were included. There were enrolled patients with ACS (STEMI, NSTEMI, unstable angina pectoris), which represent $54 \%$, as well as patients who underwent elective CAG for stable forms of IHD (46\%). In $49 \%$ of patients, selective CAG without any intervention was performed, $51 \%$ patients underwent PCI; 89\% of all interventions were performed via femoral artery, $11 \%$ via radial artery. More characteristics related to invasive procedure are displayed in Table 1 . Mean age was $69 \pm 11$ years, $64 \%$ patients were female. Other characteristics are summarized in Table 2 .

The number of bleeding complications according to localization shows that the most often bleeding occurred at access site (84.9\%) while the ratio of femoral vs. radial access in this subgroup remains the same as in global study population (89\% femoral artery and $11 \%$ radial artery). Other localizations were: intracerebral $0.9 \%$, cardiac tamponade $1.9 \%$, gastrointestinal bleeding $1.9 \%$, retroperitoneal bleeding $4.7 \%$, epistaxis $0.9 \%$, hematuria $1.9 \%$ and in $2.8 \%$ of bleeding the exact localization was not found (Table 3 ). When counting rate of bleeding according to hemodynamic 
Table 1. Procedure relate $d$ characteristics.

\begin{tabular}{lc}
\hline ACS total & $57(54 \%)$ \\
STEMI & $13(12 \%)$ \\
NSTEMI & $23(22 \%)$ \\
Unstable angina pectoris & $20(19 \%)$ \\
Ventricular fibrillation & $1(1 \%)$ \\
Non-ACS & $49(46 \%)$ \\
Stable angina pectoris & $32(30 \%)$ \\
Valvular heart disease & $9(8 \%)$ \\
Heart failure & $6(6 \%)$ \\
Other & $2(2 \%)$ \\
PCI & $52(49 \%)$ \\
CAG (without any intervention) & $54(51 \%)$ \\
Femoral access & $94(89 \%)$ \\
Radial access & $12(11 \%)$ \\
Left main coronary artery lesion & $6(5.7 \%)$ \\
Number of affected vessels: & \\
3-vessel disease & $27(25 \%)$ \\
2-vessel disease & $23(22 \%)$ \\
1-vessel disease & $20(19 \%)$ \\
Normal CAG/coronary atherosclerosis & $34(32 \%)$ \\
N/A & $2(2 \%)$ \\
Periprocedural medication: & \\
ASA & $13(12 \%)$ \\
ADP antagonists & $38(36 \%)$ \\
Heparin/LMWH & $56(53 \%)$ \\
GP Ilb/llla inhibitors & $8(8 \%)$ \\
Other & $5(5 \%)$ \\
\hline NCS & \\
\hline
\end{tabular}

ACS - acute coronary syndrome; STEMI - ST elevation myocardial infarction; NSTEMI - non-ST elevation myocardial infarction; $\mathrm{PCl}$ - percutaneous coronary intervention; CAG - coronary angiography; ASA - acetylsalicylic acid; ADP - adenosine diphosphate; LMWH - low molecular weight heparin; GP Ilb/llla - glycoprotein Ilb/lla; N/A — not available

status and consequent treatment the bleeding with no need of specific therapy represents more than a half of all bleedings $(54.7 \%), 14.2 \%$ patients needed local treatment and $2.8 \%$ had to undergo surgical procedure. Blood transfusion had to be administered in $14.2 \%$ patients. Severe complications as hemodynamic instability and hemorrhagic shock that needed intensive treatment at coronary unit appeared in $5.7 \%$, resp. $1.9 \%$. The most severe bleeding leading to death occurred in $6.6 \%$ (Table 4 ).

When TIMI classification, which relies mainly on laboratory criteria, was applied on our study population, the rate of severe bleeding complications was $9.4 \%$. Using GUSTO classification that is based mainly on clinical data, the percentage of severe bleeding complication was counted $15.1 \%$.
Table 2. Baseline characteristics.

\begin{tabular}{|c|c|}
\hline Mean age $\pm S D$ & $69.6 \pm 11$ \\
\hline \multicolumn{2}{|l|}{ Gender: } \\
\hline Male & $38(36 \%)$ \\
\hline Female & $68(64 \%)$ \\
\hline \multicolumn{2}{|l|}{ Body mass index $\left[\mathrm{kg} / \mathrm{m}^{2}\right]$ : } \\
\hline$<20$ & $2(1.9 \%)$ \\
\hline $20-25$ & $23(21.7 \%)$ \\
\hline $25-30$ & $44(41.5 \%)$ \\
\hline $30-35$ & $19(17.9 \%)$ \\
\hline $35-40$ & $11(10.4 \%)$ \\
\hline$>40$ & $4(3.8 \%)$ \\
\hline \multicolumn{2}{|l|}{ Smoking: } \\
\hline Smoker & $22(21 \%)$ \\
\hline No smoker & $59(56 \%)$ \\
\hline Ex smoker & $25(24 \%)$ \\
\hline History of IHD & $44(42 \%)$ \\
\hline Previous $\mathrm{PCl}$ & $13(12 \%)$ \\
\hline Previous CABG & $9(8 \%)$ \\
\hline Arterial hypertension & $80(75 \%)$ \\
\hline Diabetes mellitus & $32(30 \%)$ \\
\hline \multicolumn{2}{|l|}{ Treatment of diabetes mellitus: } \\
\hline Insulin therapy & $6(19 \%)$ \\
\hline Oral anti-diabetic medication & $9(28 \%)$ \\
\hline Diet & $8(25 \%)$ \\
\hline No treatment & $3(9 \%)$ \\
\hline $\mathrm{N} / \mathrm{A}$ & $6(19 \%)$ \\
\hline Dyslipidemia & $55(52 \%)$ \\
\hline Peripheral artery disease & $9(8 \%)$ \\
\hline Stroke & $8(8 \%)$ \\
\hline Atrial fibrillation & $18(17 \%)$ \\
\hline Chronic kidney disease & $71(67 \%)$ \\
\hline \multicolumn{2}{|l|}{ Chronic kidney disease stage: } \\
\hline 2 & $28(39 \%)$ \\
\hline 3 & $32(45 \%)$ \\
\hline 4 & $6(8 \%)$ \\
\hline 5 & $5(7 \%)$ \\
\hline History of bleeding & $7(7 \%)$ \\
\hline History of pulmonary embolism/DVT & $10(9 \%)$ \\
\hline Known coagulopathy & $0(0 \%)$ \\
\hline Malignity in history & $6(6 \%)$ \\
\hline
\end{tabular}

$\mathrm{SD}$ - standard deviation; IHD - ischemic heart disease;

$\mathrm{PCl}$ - percutaneous coronary intervention; CABG - coronary artery bypass grafting; DVT - deep venous thrombosis; N/A not available

The PLATO and BARC classification are more complex and take into account both clinical and laboratory data. In these cases, the incidence of 
Table 3. Number of bleeding according to localization.

\begin{tabular}{lc}
\hline Localization of bleeding & $\mathbf{N}(\%)$ \\
\hline Intracerebral & $1(0.9 \%)$ \\
Cardiac tamponade & $2(1.9 \%)$ \\
Gastrointestinal bleeding & $2(1.9 \%)$ \\
Retroperitoneal & $5(4.7 \%)$ \\
Epistaxis & $1(0.9 \%)$ \\
Hematuria & $2(1.9 \%)$ \\
Access site hematoma & $90(84.9 \%)$ \\
Unknown localization & $3(2.8 \%)$ \\
\hline
\end{tabular}

Table 4. Number of bleeding according to hemodynamic status and consequent treatment.

\begin{tabular}{lc}
\hline Type of bleeding & N (\%) \\
\hline Fatal bleeding & $7(6.6 \%)$ \\
Bleeding with hemorrhagic shock & $2(1.9 \%)$ \\
Bleeding with hemodynamic instability & $6(5.7 \%)$ \\
Bleeding with transfusions needed & $15(14.2 \%)$ \\
Surgical revision & $3(2.8 \%)$ \\
Local therapy & $15(14.25)$ \\
With no need of treatment & $58(54.7 \%)$ \\
\hline
\end{tabular}

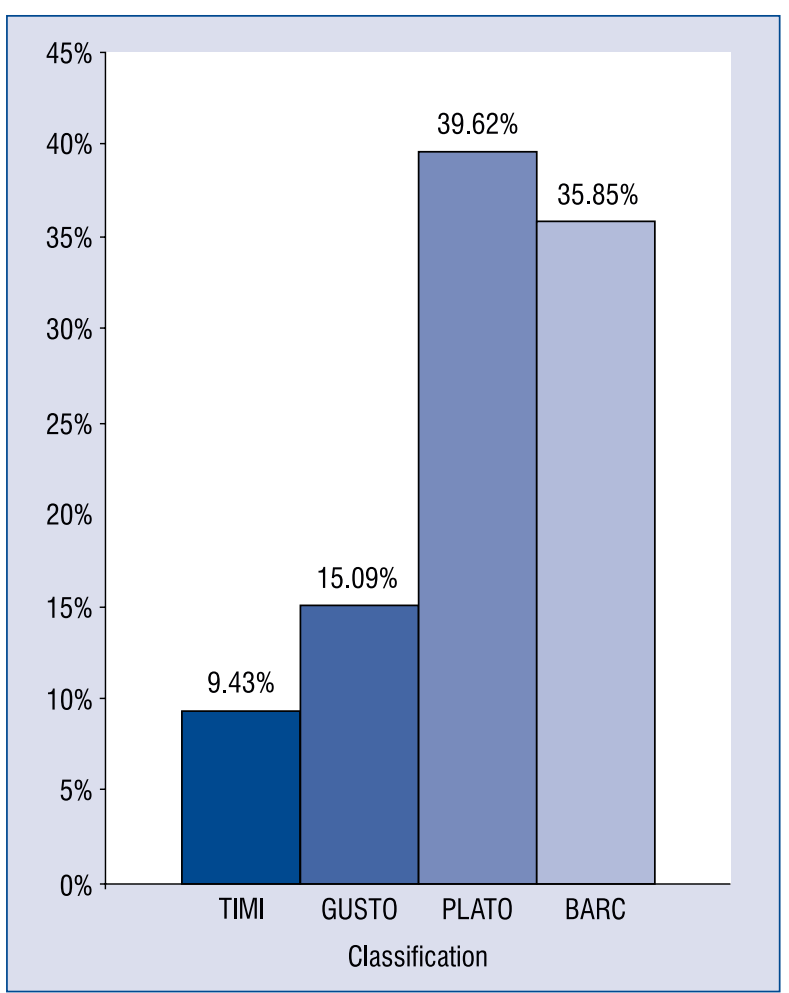

Figure 1. Number of severe bleeding complications using TIMI, GUSTO, PLATO and BARC classifications. severe bleeding was counted $39.6 \%$ for PLATO classification and $35.9 \%$ for BARC classification (Fig. 1).

Then we counted the number of severe bleeding complications according to each scale (TIMI, GUSTO, PLATO, and BARC) in all groups of patients presented with different hemodynamic changes separately. All classifications show bleeding as major (100\%) in the fatal bleeding category. At the second pole of the severity, meaning bleeding with no need of treatment or local therapy, the classifications seem to be also quite consistent. Most of them are marked as minor. In a group where no treatment was necessary, there was a minor marked $100 \%$ with TIMI classification, $100 \%$ with GUSTO, $87 \%$ with PLATO and $93 \%$ with BARC classification. In a group of patients who underwent local therapy, $100 \%$ bleeding were marked as minor with using TIMI classification, 93\% with GUSTO, $80 \%$ with PLATO and $73 \%$ with BARC classification. The results then vary depending on which classification was used in other categories. In the second severe bleeding condition with hemorrhagic shock, TIMI classification marks as severe only $50 \%$ while other 3 classifications indicate bleeding as severe in all cases. The differences are even more striking in next 3 boxes. Patients who presented with hemodynamic instability are marked as severe in $67 \%$ using BARC classifications, in $100 \%$ using PLATO and GUSTO and contrary in $83 \%$ are marked as minor when TIMI applied. Similar situation arises in a group of patients needed blood transfusion or surgical revision where TIMI and GUSTO show opposite results in a rate of major bleeding than PLATO and BARC classifications. Details are displayed in Figure 2.

Finally, we compared how hemodynamic compromise relates with each of these bleeding classifications, meaning the tightness of bounding between these 2 parameters. For that purpose the Kendall's tau-c was used and its numerical value for TIMI classification is -0.4719 (95\% CI -0.633 to -0.311$)$, for GUSTO $-0.6952(95 \%$ CI -0.827 to $-0,564)$, for PLATO $-0.6520(95 \%$ CI -0.776 to -0.528$)$ and for BARC -0.5991 (95\% CI -0.701 to -0.500$)$ using their extended version. When it is applied to the merged version of bleeding classifications focusing explicitly to serious bleeding complications, the value for TIMI is -0.4106 (95\% CI -0.599 to -0.222 ), for GUSTO -0.5355 (95\% CI -0.718 to -0.353$)$, for PLATO -0.5513 (95\% CI -0.729 to -0.374$)$ and for BARC -0.7552 (95\% CI -0.897 to -0.612$)$. The strongest association 


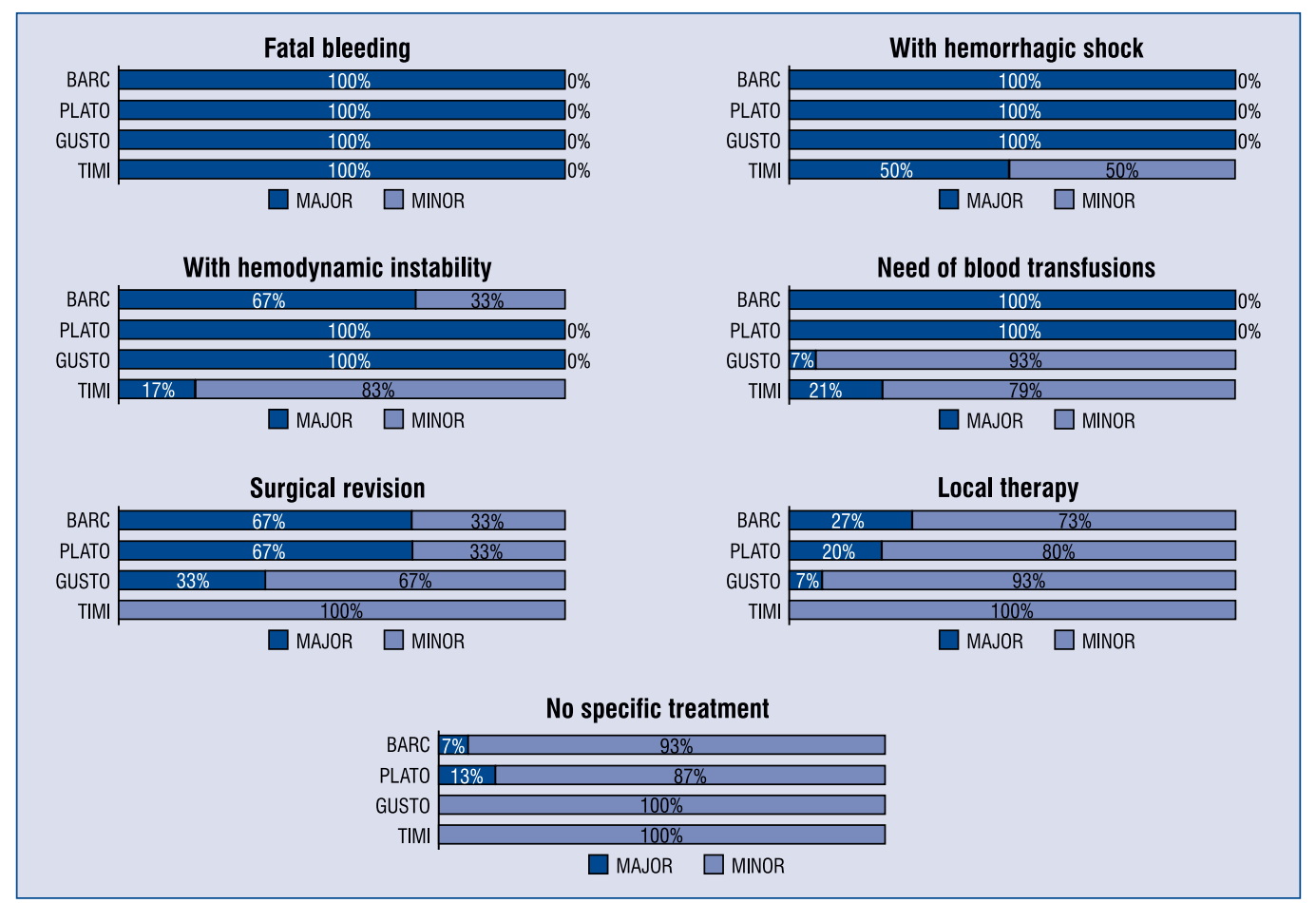

Figure 2. Comparison of bleeding according to hemodynamic status and rate of major bleeding of each bleeding classifications.

with the hemodynamic compromise was found for merged version of BARC classification and also the 95\% confidence interval (CI) shows the smallest variability in BARC classification (in both cases comparing extended or merged version).

\section{Discussion}

Our results show significant differences in number of severe bleeding complications that may lead to huge heterogeneity in presentation of outcomes when different classifications are used and that classifications do not always reflect the real hemodynamic compromise of the patient and so it could underestimate the real risk. The differences are emphasized mainly for those bleeding complications that are classified in the middle part of the scale (meaning not life-threatening or minimal).

When focused on localization (Table 3 ) it is obvious that the greatest number of bleeding complications appeared at access site (85\%). The evaluation of influence on hemodynamic status in the same group of patients shows more equal stratification (Table 4). These findings suggest that even access site bleeding may result in very serious condition with fatal outcome. Even if 55\% of patients did not need any specific treatment at the moment, the bleeding may prolong their hospitalization, which in turn may bring many other complications leading to increased morbidity and mortality [18]. Also, administration of blood transfusion, which occurred in $14.2 \%$ of patients, is associated with higher rate of morbidity and mortality and it is not recommended as routinely as before [19]. All these factors mentioned above contribute to worse short-term, as well as longterm prognosis for all patients who demonstrate bleeding. Therefore bleeding, as an indicator of safety of all newly introduced procedures and drugs affecting hemostasis in cardiology, is very preciously observed and reported in all clinical trials using different bleeding classifications.

The first formal classification of bleeding complications associated to treatment of patients with ACS was introduced in 1988 as a part of the TIMI trial [14]. The TIMI classification is based mainly on laboratory criteria. The second widespread classification was introduced in GUSTO trial in 1993 [15]. This classification, compared to TIMI classification, relies mainly on clinical outcomes. These 2 classifications, more or less modified, have been used in many clinical trials [20,21]. Their limitations seem to be in preferable use of pure laboratory or pure clinical criteria. This is the 
reason why numbers of different classifications, combining advantages of both, have been developed since then [22-24]. Specific classification for evaluation of bleeding complications, containing combination of clinical and laboratory criteria, was then estimated for PLATO trial [12]. Supplementary Table S1 displays detailed definition of these classifications. It is obvious that in that wide range of classifications it is almost impossible to compare clinical outcomes across executed studies [25]. In response to this situation, the tendency of establishing one standardized classification has arisen [26]. After a few attempts earlier, finally in 2011, BARC, a group of independent experts summarized all known evidence and data from clinical trials and evaluated new standardized bleeding classification for cardiovascular clinical trials (Supplementary Table S2) [27]. This classification seems to be comprehensive enough and meets all criteria for comfortable general use but was not proven yet in a clinical practice [16].

From wide range of the bleeding classifications that are normally used in large randomized clinical trials we applied 4 of them in our group of patients. Using 4 different classifications (TIMI, GUSTO, PLATO and BARC) we observed percentage of severe bleeding complications, as for better comparability we merged categories of severe bleeding in PLATO and BARC classification into one group. The results after application of these 4 different classifications scales on one group of patients show significant differences between incidences of severe periprocedural bleeding complications depending on classification used. The rates of severe bleeding complication were significantly lower when the simplest classifications, TIMI and GUSTO, were applied (9.4\% and $15.1 \%$, respectively). Using more complex classification that is in our study represented by classification from PLATO trial, the rate of severe bleeding complications increases almost 4-times (39.6\%). Similar percentage of severe complication which was reached with PLATO classification was also achieved with BARC classification (35.9\%). Except for finding that outcomes may be strongly influenced with selected classification, these data may indicate that using simpler and limited classification (using pure laboratory or pure clinical data) could underestimate the number of severe bleeding complications. These data also support current recommendation for all clinicians and researchers to use one standardized bleeding classification. When comparing these classifications in their original extended version, BARC classification seems to be the most systematic, complex and thus the most suitable for that purpose.

A clinical trial comparing TIMI and GUSTO classification proved that bleeding complications assessed with clinical criteria (GUSTO) show to be more important than those assessed by laboratory criteria (TIMI) in terms of clinical outcomes (death/ /myocardial infarction) [13]. These findings led us to assume that the real hemodynamic compromise caused by bleeding may be an approximate indicator of potential dangerousness of each bleeding episode. So we counted a number of severe bleeding complications separately (using 4 classifications above) in each category of hemodynamic changes due to bleeding. With this analysis we found even bigger diversity in outcomes resulting from the types of classification was used. At two opposite poles, meaning the most serious clinical conditions resulting in deaths on the first pole and the bleeding with no need of treatment or with a need of local therapy on the second pole, the results in number of major vs. minor bleeding show similar incidence in all used classification. Meanwhile, the differences are rising rapidly in the middle part of the notional scale. But even when very serious clinical conditions appeared with an intensive coronary unit treatment (hemorrhagic shock and hemodynamic instability caused by bleeding) the data resulting from using different classifications led to significantly different and sometimes almost opposite outcomes (Fig. 2).

Therefore, finally, we measured the power of association between the real clinical and hemodynamic status and the severity of bleeding defined by each classification. The results show that in original extended version of all classifications the association is found to be strongest for GUSTO classification and the weakest for TIMI. The strong association in GUSTO classification is expected and can be explained using pure clinical data in this classification (the same as the weakest association in TIMI classification that does not reflect clinical statement at all). However, when the same measurement of association is applied on the merged version of bleeding classifications (reflecting all severe bleedings in one group) the strongest correlation with the real clinical condition is found when using BARC classification. The weakest association remains using TIMI classification.

\section{Limitations of the study}

There are several limitations that should be addressed. The major limitation is the small sample size with small number of patients with 
hemodynamic instability, shock or fatal bleeding, but according to low rate of bleeding in general, the appearance of bleeding complications, particularly the most serious, is very low. In one of the most recent clinical trial, ACCOAST trial, where only high risk patients with ACS were enrolled, composite of major and minor TIMI bleeding in a control group occurred in $1.2 \%$, life-threatening bleeding occurred in $0.51 \%$ and no fatal bleeding was observed [28]. If we anticipated the total number of patients who underwent CAG/PCI in our work according to data above, it would match almost 9,000 examined patients. As we evaluated both patients with ACS, as well as those with stable form of IHD, and as in most participating centers the radial access is preferred, the incidence of bleeding is assumed to be even lower.

Secondly, the study population included both patients undergoing elective invasive procedure and patients with ACS. According to this limitation, we cannot estimate the incidence of bleeding and identify any risk factors of bleeding events. However, the purpose of the study was to prove the utility and correlation of different bleeding classifications in real clinical practice, not to identify risk factors for bleeding.

\section{Conclusions}

All these findings demonstrate how different classification may strongly affect outcomes from clinical trials and then subsequently strongly influence the recommendations for evidence based treatment of patients with IHD. One unified classification that could be used generally in practice and across clinical trials in cardiology seems to be necessary. The classification must be constructed to capture all bleeding events that are important and meaningful as for patients as for clinical outcomes while remaining simple, broadly applicable and easy for use. Recently introduced BARC classification seems to meet all those criteria for applicability in routine clinical and research practice. In that case, all researchers, experts and clinicians should be aware of this new classification and report bleeding events according to this BARC definition so it could be proven in practice and the data can be comparable in the future.

Funding: This work was supported by the Third Faculty of Medicine, Charles University, Prague, Research Project UNCE204010 and Research Project PRVOUK P35.

Conflict of interest: None declared

\section{References}

1. Fox KA, Steg PG, Eagle KA et al. Decline in rates of death and heart failure in acute coronary syndromes, 1999-2006. JAMA, 2007; 297: 1892-1900.

2. Collaborative meta-analysis of randomised trials of antiplatelet therapy for prevention of death, myocardial infarction, and stroke in high risk patients. BMJ, 2002; 324: s71-s86.

3. Batchelor WB, Anstrom KJ, Muhlbaier LH et al. Contemporary outcome trends in the elderly undergoing percutaneous coronary interventions: results in 7,472 octogenarians. National Cardiovascular Network Collaboration. J Am Coll Cardiol, 2000; 36: 723-730.

4. Fox KA, Carruthers K, Steg PG et al. Has the frequency of bleeding changed over time for patients presenting with an acute coronary syndrome? The global registry of acute coronary events. Eur Heart J, 2010; 31: 667-75.

5. Eikelboom JW, Mehta SR, Anand SS, Xie C, Fox KA, Yusuf S. Adverse impact of bleeding on prognosis in patients with acute coronary syndromes. Circulation, 2006; 114: 774-782.

6. Doyle BJ, Ting HH, Bell MR et al. Major femoral bleeding complications after percutaneous coronary intervention: incidence, predictors, and impact on long-term survival among 17,901 patients treated at the Mayo Clinic from 1994 to 2005. J Am Coll Cardiol Cardiovasc Interv, 2008; 1: 202-209.

7. Segev A, Strauss BH, Tan M, Constance C, Langer A, Goodman SG Predictors and 1-year outcome of major bleeding in patients with non-ST-elevation acute coronary syndromes: insights from the Canadian Acute Coronary Syndrome Registries. Am Heart J, 2005; 150: 690-694.

8. Lauer MA, Karweit JA, Cascade EF, Lin ND, Topol EJ. Practice patterns and outcomes of percutaneous coronary interventions in the United States: 1995 to 1997. Am J Cardiol, 2002; 89: 924-929.

9. Kinnaird TD, Stabile E, Mintz GS et al. Incidence, predictors, and prognostic implications of bleeding and blood transfusion following percutaneous coronary interventions. Am J Cardiol, 2003; 92: 930-935.

10. Fuchs S, Kornowski R, Teplitsky I et al. Major bleeding complicating contemporary primary percutaneous coronary interventions-incidence, predictors, and prognostic implications. Cardiovasc Revasc Med, 2009; 10: 88-93.

11. Antman EM, Wiviott SD, Murphy SA et al. Early and late benefits of prasugrel in patients with acute coronary syndromes undergoing percutaneous coronary intervention: A TRITON-TIMI 38 (TRial to Assess Improvement in Therapeutic Outcomes by Optimizing Platelet InhibitioN with Prasugrel-Thrombolysis In Myocardial Infarction) analysis. J Am Coll Cardiol, 2008; 51: 2028-2033.

12. Becker RC, Bassand JP, Budaj A et al. Bleeding complications with the P2Y12 receptor antagonists clopidogrel and ticagrelor in the PLATelet inhibition and patient Outcomes (PLATO) trial. Eur Heart J, 2011; 32: 2933-2944.

13. Rao SV, O'Grady K, Pieper KS et al. A comparison of the clinical impact of bleeding measured by two different classifications among patients with acute coronary syndromes. J Am Coll Cardiol, 2006; 47: 809-816.

14. Rao AK, Pratt C, Berke A et al. Thrombolysis in Myocardial Infarction (TIMI) Trial: Phase I: Hemorrhagic manifestations and changes in plasma fibrinogen and the fibrinolytic system in patients treated with recombinant tissue plasminogen activator and streptokinase. J Am Coll Cardiol, 1988; 11: 1-11. 
15. The GUSTO Investigators. An international randomized trial comparing four thrombolytic strategies for acute myocardial infarction. N Engl J Med, 1993; 329: 673-682.

16. Steg PG, Huber K, Andreotti F et al. Bleeding in acute coronary syndromes and percutaneous coronary interventions: Position paper by the Working Group on Thrombosis of the European Society of Cardiology. Eur Heart J, 2011; 32: 1854-1864.

17. Göktaş A, İşçi Ö. A Comparison of the most commonly used measures of association for doubly ordered square contingency tables via simulation. Metodološki Zvezki, 2011; 8: 17-37.

18. Vavalle JP, Lopes RD, Chen AY et al. Hospital length of stay in patients with non-ST-segment elevation myocardial infarction. Am J Med, 2012; 125: 1085-1094.

19. Rao SV, Jollis JG, Harrington RA et al. Relationship of blood transfusion and clinical outcomes in patients with acute coronary syndromes. JAMA, 2004; 292: 1555-1562.

20. The PARAGON Investigators. International, randomized, controlled trial of lamifiban (a platelet glycoprotein IIb/IIIa inhibitor), heparin, or both in unstable angina. Platelet IIb/IIIa Antagonism for the Reduction of Acute coronary syndrome events in a Global Organization Network. Circulation, 1998; 97: 2386-2395.

21. Ferguson JJ, Califf RM, Antman EM et al. Enoxaparin vs unfractionated heparin in high-risk patients with non-ST-segment elevation acute coronary syndromes managed with an intended early invasive strategy: Primary results of the SYNERGY randomized trial. JAMA, 2004; 292: 45-54.

22. Mehta SR, Yusuf S. The Clopidogrel in Unstable angina to prevent Recurrent Events (CURE) trial programme; rationale, de- sign and baseline characteristics including a meta-analysis of the effects of thienopyridines in vascular disease. Eur Heart J, 2000; 21: 2033-2041.

23. Mehta SR, Bassand JP, Chrolavicius S et al. Design and rationale of CURRENT-OASIS 7: A randomized, $2 \times 2$ factorial trial evaluating optimal dosing strategies for clopidogrel and aspirin in patients with ST and non-ST-elevation acute coronary syndromes managed with an early invasive strategy. Am Heart J, 2008; 156: 1080-1088e1.

24. Stiles MK, Dabbous OH, Fox KA. Bleeding events with antithrombotic therapy in patients with unstable angina or non-ST-segment elevation myocardial infarction; insights from a large clinical practice registry (GRACE). Heart Lung Circ, 2008; 17: 5-8.

25. The PURSUIT Trial Investigators. Inhibition of platelet glycoprotein IIb/IIIa with eptifibatide in patients with acute coronary syndromes. Platelet Glycoprotein IIb/IIIa in Unstable Angina: Receptor Suppression Using Integrilin Therapy. N Engl J Med, 1998; 339: 436-443.

26. Rao SV, Eikelboom J, Steg PG et al. Standardized reporting of bleeding complications for clinical investigations in acute coronary syndromes: A proposal from the academic bleeding consensus (ABC) multidisciplinary working group. Am Heart J, 2009; 158: 881-886e1.

27. Mehran R, Rao SV, Bhatt DL et al. Standardized bleeding definitions for cardiovascular clinical trials: a consensus report from the Bleeding Academic Research Consortium. Circulation, 2011; 123: 2736-2747.

28. Montalescot G, Collet JP, Ecollan P et al. Effect of prasugrel pretreatment strategy in patients undergoing percutaneous coronary intervention for NSTEMI: The ACCOAST-PCI study. J Am Coll Cardiol, 2014; 64: 2563-2571.

Supplementary Table S1. TIMI, GUSTO and PLATO bleeding classifications.

\begin{tabular}{|c|c|c|c|}
\hline & Major & Minor & Minimal \\
\hline \multirow[t]{2}{*}{ TIMI } & $\begin{array}{l}\text { Fatal, intracranial or associated } \\
\text { with a decrease in hemoglobin } \\
>5 \mathrm{~g} / \mathrm{dL} \text { (or } 15 \% \text { in hematocrit) }\end{array}$ & $\begin{array}{l}\text { Hemoglobin decreasing } \\
>3 \mathrm{~g} / \mathrm{dL} \text { (or hematocrit } \\
\text { decreasing at least } 10 \% \text { ) }\end{array}$ & Any other bleeding \\
\hline & Severe & Moderate & Mild \\
\hline \multirow[t]{2}{*}{ GUSTO } & $\begin{array}{l}\text { Intracerebral or if it resulted in } \\
\text { substantial hemodynamic } \\
\text { compromise requring treatment }\end{array}$ & Need for transfusion & $\begin{array}{l}\text { Other bleeding, not requiring } \\
\text { transfusion or causing } \\
\text { hemodynamic compromise }\end{array}$ \\
\hline & Fatal/life-threatening & Major & Minor \\
\hline PLATO & $\begin{array}{l}\text { Fatal bleeding, intrapericardial } \\
\text { bleeding with cardiac tampon- } \\
\text { ade, intracranial bleeding, severe } \\
\text { hypotension, hypovolemic shock } \\
\text { due to bleeding, decline in } \\
\text { hemoglobin of } 5.0 \mathrm{~g} / \mathrm{dL} \text {, need for } \\
\text { transfusion more than } 4 \text { units }\end{array}$ & $\begin{array}{l}\text { Clinical significant disability, } \\
\text { drop of hemoglobin } 3-5 \mathrm{~g} / \mathrm{dL} \text {, } \\
\text { requiring } 2-3 \text { units red blood } \\
\text { cell transfusion }\end{array}$ & $\begin{array}{l}\text { Any bleeding event requiring } \\
\text { medical intervention but not } \\
\text { meeting the criteria for major } \\
\text { bleeding }\end{array}$ \\
\hline
\end{tabular}


Supplementary Table S2. BARC bleeding classification.

\begin{tabular}{|c|c|}
\hline Type & Description \\
\hline 0 & No bleeding \\
\hline 1 & $\begin{array}{l}\text { Bleeding that is not actionable and does not cause the patient to seek unscheduled performance } \\
\text { of studies, hospitalization, or treatment by a health care professional }\end{array}$ \\
\hline 2 & $\begin{array}{l}\text { Any overt, actionable sign of hemorrhage (e.g. more bleeding than would be expected for a clinical } \\
\text { circumstance; including bleeding found by imaging alone) that does not fit the criteria for type } 3,4 \text {, } \\
\text { or } 5 \text {, but does met at least one of the following criteria: (1) requiring non-surgical, medical intervention } \\
\text { by a health care professional, (2) leading to hospitalization or increased level of care, (3) prompting } \\
\text { evaluation }\end{array}$ \\
\hline \multicolumn{2}{|l|}{3} \\
\hline \multirow[t]{2}{*}{$3 a$} & Overt bleeding plus hemoglobin drop of 3 to $5^{*} \mathrm{~g} / \mathrm{dL}$ (provided hemoglobin drop is related to bleed) \\
\hline & Any transfusion with overt bleeding \\
\hline \multirow[t]{4}{*}{$3 b$} & Overt bleeding plus hemoglobin drop $\geq 5^{*} \mathrm{~g} / \mathrm{dL}$ (provided hemoglobin drop is related to bleed) \\
\hline & Cardiac tamponade \\
\hline & Bleeding requiring surgical intervention for control (excluding dental/nasal/skin/hemorrhoid) \\
\hline & Bleeding requiring intravenous vasoactive drugs \\
\hline \multirow[t]{3}{*}{$3 c$} & $\begin{array}{l}\text { Intracranial hemorrhage (does not include microbleeds or hemorrhagic transformation; } \\
\text { does include intraspinal) }\end{array}$ \\
\hline & Subcategories; confirmed by autopsy or imaging or lumbar puncture \\
\hline & Intra-ocular bleed compromising vision \\
\hline \multirow{5}{*}{$\begin{array}{l}\text { 4: CABG- } \\
\text {-related } \\
\text { bleeding }\end{array}$} & Perioperative intracranial bleeding within $48 \mathrm{~h}$ \\
\hline & Reoperation following closure of sternotomy for the purpose of controlling bleeding \\
\hline & Transfusion of $\geq 5$ units of whole blood or packed red blood cells within a $48 \mathrm{~h}$ period $\mathrm{d}^{* *}$ \\
\hline & Chest tube output $\geq 2 \mathrm{~L}$ within a $24 \mathrm{~h}$ period \\
\hline & $\begin{array}{l}\text { If a CABG-related bleed is not adjudicated as at least a type } 3 \text { severity event, it will be classified } \\
\text { as 'not a bleeding event' }\end{array}$ \\
\hline \multicolumn{2}{|l|}{$\begin{array}{l}\text { 5: fatal } \\
\text { bleeding }\end{array}$} \\
\hline $5 a$ & Probable fatal bleeding: no autopsy or imaging confirmation, but clinically suspicious \\
\hline $5 b$ & Definite fatal bleeding: overt bleeding or autopsy or imaging confirmation \\
\hline
\end{tabular}

Platelet transfusions should be recorded and reported, but are not included in these definitions until further information is obtained about the relationship to outcomes; *Corrected for transfusion ( $1 \mathrm{U}$ packed red blood cells or $1 \mathrm{U}$ whole blood $=1 \mathrm{~g} / \mathrm{dL}$ hemoglobin); ** Only allogeneic transfusions are considered transfusions for BARC type 4 bleeding. Cell saver products will not be counted; CABG - coronary artery bypass grafting 